\title{
Alert System for High Speed and Acceleration in Land Vehicles
}

\author{
Mario A. Gomez N. ${ }^{1}$, Julian R. Camargo L. ${ }^{2}$ and Cesar A. Perdomo Ch${ }^{2^{*}}$ \\ 'Wavecomm Corporation, Bogota D.C., Colombia; mgomez@wavecomm.net \\ 2Universidad Distrital Francisco Jose de Caldas, Bogota D.C., Colombia; jcamargo@udistrital.edu.co, \\ cperdomo@udistrital.edu.co
}

\begin{abstract}
Objectives: Design and build an electronic audiovisual warning device for land transport vehicles operators which allows correcting, in real time, operation errors for overstep the speed limits and/or acceleration established. Additionally, the device allows the identification by keyboard of the vehicle operator for the subsequent generation of individualized statistics. Methods/Analysis: This investigation will allow the development of an electronic equipment that alerts the land vehicles operator, in real time and at the moment when they are getting out of control in some of the critical variables for safe driving, namely speed and acceleration, allowing to correct the mistakes, thanks to a timely and easy to understand information. Findings: The incorporation of electronic early warning devices of common driving errors for land transport operators allows a significant reduction in road accident rates, thanks to the stimulation of self-care and self-control, by means of agile and timely information within the vehicle cabin. Improvements: Currently the major research in this subject is around the training and investigation of accidents but do not have many objective tools to measure and qualify operators in the application of the standards and improvement plans that are taught (industrial safety, basic driving, traffic regulations, defensive driving and basic mechanics, among others).
\end{abstract}

Keywords: Accelerometers, Automotive Accident Prevention, MEMS, Microcontroller, Road Safety, Speedometer

\section{Introduction}

The driving activity of land vehicles is one of the most common and necessary tasks for the correct development of the economy in today's society. Transporting yourself to your workplaces, studios, hospitals, shopping centers, resorts, among others, would be almost impossible for most people without some kind of vehicle, but not too many people know that this activity is classified like an activity of highest risk, accident and mortality rate according to the World Health Organization (WHO) ${ }^{1}$. In Colombia alone, the transport ministry annually reports more than 200,000 traffic accidents, resulting in nearly 5,000 fatalities and more than 40,000 seriously injured ${ }^{2}$.
Few things have been done to prevent the appearance of those moments of deconcentration, which result in catastrophic and deadly consequences on the world's roads, in part because any additional restrictions, such as raising the safety standards for vehicles or increase the conditions to obtain and maintain a driving license, would directly impact the growth of the automotive sector, one of the most prosperous and with the greatest influence on global political and economic activity. Some efforts of vehicle manufacturers have taken place in the industry, many of them aimed at mitigating the consequences before the imminent appearance of accidents, rather than preventing them.

*Author for correspondence 
Some statistics that motivate this work are really terrifying, since approximately 1.2 million people die every year in the world for causes related to traffic accidents and close to 50 million are seriously injured, most of these accidents are related to speeding, driving in a drunkenness, lack of expertise in the handling of accelerations and decelerations of the vehicle, distractions with cell phones, misuse of the seatbelt and fatigue.

These numbers are much more worrying when are compared to the annual number of deaths worldwide in the same period, due to other common causes of death such as AIDS (1.8 million), alcoholism (2.2 million), tuberculosis (1.1 million), tobacco (5 million)), cholera $(374,000)$ and malaria $(650,000)$, which indicates that we are really facing a public health problem, which according to the World Health Organization, tends to get worse in the next 15 years $^{2}$.

\section{Time, Distance, Speed and Acceleration, the Keys of the Control of a Vehicle}

When the task of designing a controller is undertaken, it is absolutely necessary to have a very good mathematical model of the process. For the particular case of land vehicles, describing a mathematical model of the basic kinematic aspects that surround it, namely position, velocity and acceleration, will be taking on in this section. For this, the speed of the wheels and the speed of the vehicle will be used as state variables; additionally the torque applied to the wheel by the motor/transmission assembly will be the input variable.

The two state variables of this model will be associated to the dynamics of a wheel in rotation and the dynamic of linear displacement of the vehicle, that is, the problem will be analyzed in two dimensions for simplicity of the model. The state equations will be the result of applying Newton's laws to the wheel and the vehicle. In this way we can express the angular movement of the wheel with the Equation 1:

$$
\dot{\omega}_{W}=\frac{\left[T_{e}-T_{b}-R_{w} F_{t}-R_{w} F_{w}\right]}{J_{w}}
$$

Where $\mathrm{J}_{\mathrm{w}}$ is the moment of inertia of the wheel, $\dot{\omega}_{\mathrm{w}}$ is the angular velocity of the wheel, $\mathrm{R}_{\mathrm{w}}$ is the radius of the wheel, $\mathrm{N}_{v}$ is the normal force from the ground, $\mathrm{T}_{e}$ is the torque of the motor, $T_{b}$ is the brake torque, $F_{t}$ is the pulling force and $\mathrm{F}_{\mathrm{w}}$ is the friction of the wheel. The description above can be represented graphically in Figure 1.

The coefficient of adhesion, which is the ratio between the traction/braking force and the normal force, depends to a large extent on the wheel/ground conditions and the value of the sliding deformation coefficient $\lambda$.

In this way, the vehicles own dynamics can be analyzed in greater detail, which can be described with the Equation 2 when the vehicle is in motion:

$$
\dot{V}=\left[N_{w} F_{t}-F_{v}\right] / M_{v}
$$

$\mathrm{F}_{v}$ is the force of drag or friction due to the wind, which is a function of the speed of the vehicle, $M_{v}$ is the effective mass of the vehicle, $\mathrm{N}_{w}$ is the number of traction wheels during acceleration or the total number of wheels during braking and $\mathrm{F}_{t}$ is the average of the frictional force on the traction wheels during acceleration or the average of the friction force on all wheels during braking. Thus the linear acceleration of the vehicle will be the result of the difference between the total traction force available on the wheel/road contact surface minus the force of wind drag on the vehicle body, all this divided into the mass of the vehicle, above can be represented in Figure 2.

The total equation of the system can be written in the form of state variables, defining said variables for the expressions found for the dynamics of the wheel and the vehicle as follows:

$$
\begin{aligned}
& x_{1}=V / R_{w} \\
& x_{2}=\omega_{w}
\end{aligned}
$$

And denoting $x=\operatorname{Max}\left(x_{1}, x_{2}\right)$ you get:

$$
\begin{gathered}
\dot{x}_{1}=-f_{1}\left(\dot{x}_{1}\right)+b_{1 N} \mu(\lambda) \\
x_{2}=-f_{2}\left(x_{2}\right)-b_{2 N} \mu(\lambda)+b_{3}
\end{gathered}
$$

Where:

$$
\begin{aligned}
& T=T_{e}-T_{b} \\
& \lambda=\frac{x_{2}-x_{1}}{x}
\end{aligned}
$$




$$
\begin{gathered}
f_{1}\left(x_{1}\right)=\left[F_{v}\left(R_{w} x_{1}\right)\right] /\left(M_{v} R_{w}\right) \\
b_{1 N}=N_{v} N_{w} /\left(M_{v} R_{w}\right) \\
f_{2}\left(x_{2}\right)=F_{w}\left(x_{2}\right) / J_{w} \\
b_{2 N}=R_{w} N_{v} / J_{w} \\
b_{3}=1 / J_{w}
\end{gathered}
$$

The complete dynamic system is represented in Figure 3, where the input to the system is the torque applied to the wheels, which is the difference between the torque transmitted from the motor/transmission assembly by means of the shaft and the torque exerted, by the system of brakes. During a period of acceleration, the force transmitted by the motor is the main input, while in a deceleration period it is the torque applied by the brake system that governs the behavior of the system

Because the wheel slip is a very influential variable in the behavior of the dynamics of the vehicle under braking conditions, especially in the amount of braking force between the wheel and the ground, it is chosen as a controlled variable in the development of the braking algorithms. In some way, if you could manage to control the wheel slip, you can then easily control the braking force to get the right output to not lose control of the vehicle. In order to develop this strategy, we will have to obtain the dynamic equations of the system in terms of wheel slip.

Once you have the mathematical definition of the combined systems (Wheel - Vehicle), you could quickly go to the design of the controller, however it is necessary to evaluate in the first instance the stability of the system, since there have been found non-linearities, so the stability of the system in the linear segments should be evaluated. Mathematically, the local stability of a nonlinear system can be evaluated, linearizing the system around its equilibrium point.

The equilibrium $\mathrm{x}_{0}$ is asymptotically stable if all the eigenvalues of Df $\left(x_{0}\right)$ have strictly negative real parts. And it is unstable if Df $\left(\mathrm{x}_{0}\right)$ has eigenvalues with a positive real part. As a result, the linearization process can provide sufficient conditions for the stability of a nonlinear system over a small region near the equilibrium point, only if the system is invariant over time. Only in the case where $\mathrm{Df}\left(\mathrm{x}_{0}\right)$ has purely imaginary eigenvalues, nonlinear methods would have to be used to evaluate the stability of the system.

The non-linear equations of the vehicle have been linearized in the nearness of the equilibrium point in order to analyze the stability of the system. In this way the equilibrium point $\left(\mathrm{x}_{10}, \mathrm{x}_{20}\right)$ of the vehicular system described by the previous equations can be obtained by matching the right parts of equations (4a) and (4b) to 0 , only then can the Jacobian matrices be evaluated to analyze the stability of the system. For the deceleration condition that is one of the most important conditions of this study, the Jacobian matrix in equilibrium is shown in Equation 6:

$$
A=\left[\begin{array}{c}
-\frac{d f_{1}}{d x_{1}}\left(x_{10}\right)-b_{1 N} \frac{\delta \mu}{\delta \lambda}\left(x_{10}, x_{20}\right) \frac{x_{20}}{x_{10}^{2}} \\
b_{2} N \frac{\delta \mu}{\delta \lambda}\left(x_{10}, x_{20}\right) \frac{x_{20}}{x_{10}^{2}} \\
b_{1 N} \frac{\delta \mu}{\delta \lambda}\left(x_{10}, x_{20}\right) \frac{1}{x_{10}} \\
-\frac{d f_{2}}{d x_{2}}\left(x_{20}\right)-b_{2 N} \frac{\delta \mu}{\delta \lambda}\left(x_{10}, x_{20}\right) \frac{1}{x_{10}}
\end{array}\right]
$$

The eigen values for $A$ are obtained by solving the equation for $\lambda_{e}$

$$
\operatorname{det}\left(\lambda_{e} I-A\right)=0
$$

The real part of the eigenvalues of a are calculated as shown in Equation 8:

$$
\frac{\frac{d f_{1}}{d x_{1}}\left(x_{10}\right)+\frac{d f_{2}}{d x_{2}}\left(x_{20}\right)+\frac{\delta \mu}{\delta \lambda}\left(x_{10}, x_{20}\right)\left[b_{1 N} \frac{x_{20}}{x_{10}}+b_{2 N} \frac{1}{x_{10}}\right]}{2}
$$

Therefore $\mathrm{df}_{1} / \mathrm{dx}_{1}, \mathrm{df}_{2} / \mathrm{dx}_{2}, \mathrm{x}_{1}, \mathrm{x}_{2}, \mathrm{~b}_{1 \mathrm{~N}}$ and $\mathrm{b}_{2 \mathrm{~N}}$ are all positive, so when $\delta \lambda / \delta \mu$ is positive, the eigenvalues of $\mathrm{A}$ have a real negative part. In a complementary manner when $\delta \lambda / \delta \mu$ is negative, the eigenvalues of A have a positive real part.

$$
b_{1 N} \frac{x_{20}}{x_{10}^{2}}+b_{2 N} \frac{1}{x_{10}}>\frac{\frac{d f_{1}}{d x_{1}}\left(x_{10}\right)+\frac{d f_{2}}{d x_{2}}\left(x_{20}\right)}{\left|\frac{\delta \mu}{\delta \lambda}\right|}
$$

Therefore and under the previous condition the system is unstable $e^{4}$. 


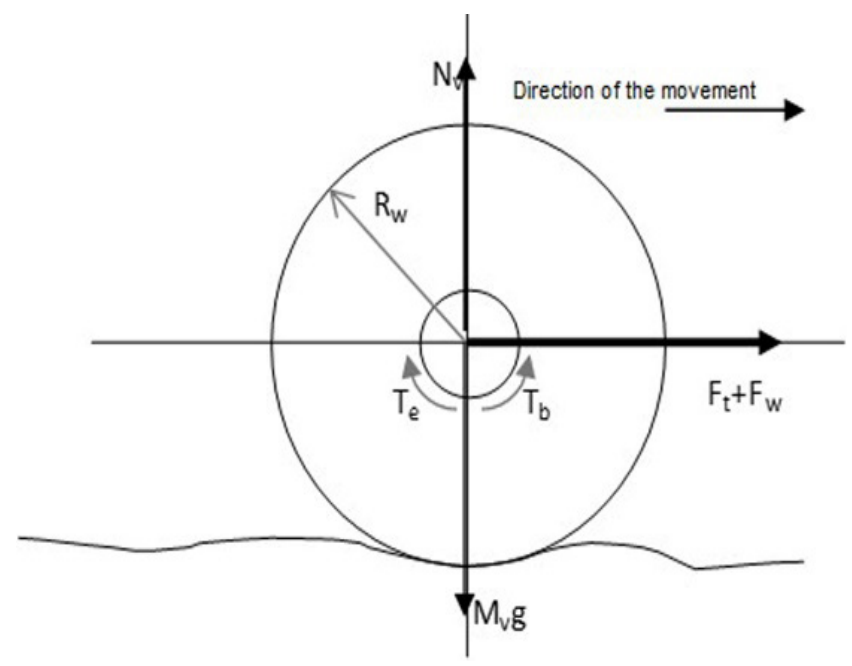

Figure 1. Representation of forces acting on a wheel.

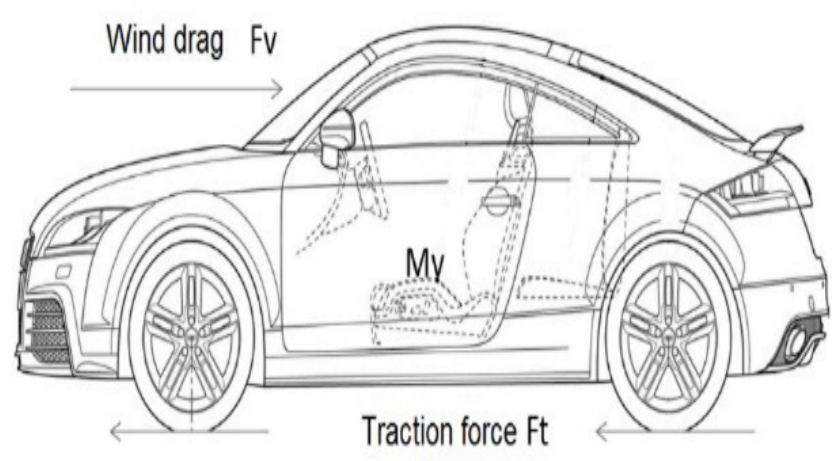

Figure 2. Dynamics of a moving vehicle (Modified from ${ }^{3}$ ).

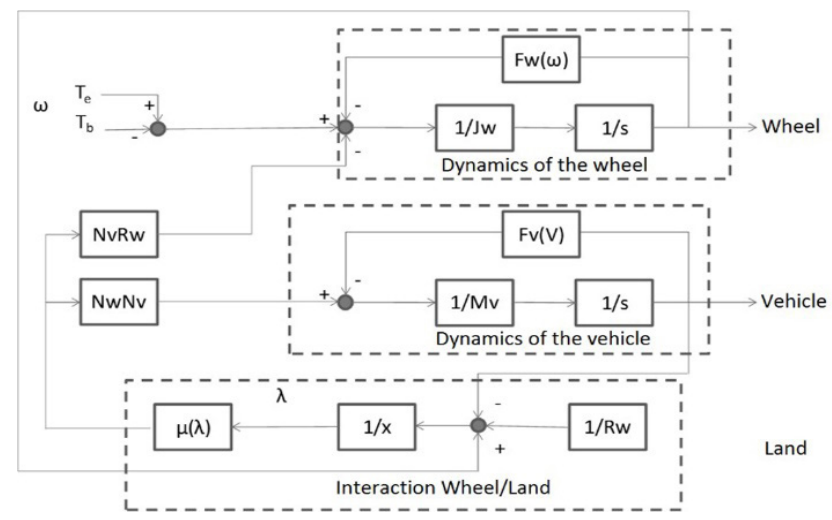

Figure 3. Block diagram of the complete dynamic system.

\section{Speed and Acceleration Sensors available in the Automotive Market}

\subsection{Electromechanical Sensors}

The first mechanical speedometer, also known as a tongue speedometer or parasitic current velocity meter (Eddy Current Speedometer), was developed by Otto Schulze, a German inventor, who was the one who registered the first patent for this type of instrument in 1902. Schulze conceived it to cover a growing need in an automotive market that was becoming increasingly popular. The speed measurement of a vehicle under the Schulze principle is based on the measurement of the rotational speed of the tires or failing that, the transmission of the vehicle, for its subsequent sending and viewing by means of an analog clock. In most commercial vehicles, the measurement is made through the transmission, where its rotational movement is coupled by means of some pinions to a braided steel cable, which due to its helical composition and design of its plastic sheath, allows flexibility and rotation through an irregular route to the dashboard of the vehicle where the active element of the speedometer is located, where really the measurement of the speed takes place ${ }^{5}$.

Inside the clock of the speedometer is a series of couplings, which by means of a spiral gear, connect the steel cable to a permanent magnet that is placed inside a metal passenger compartment known as "Speedcup" This chain of mechanisms converts the rotation of the cable into a series of impulses that induce energy to a needle by means of the permanent magnet, which deflects according to the amount of energy induced, which in turn is directly proportional to the rotation of the transmission/wheel and that according to its construction and the type of vehicle its deflection angle can vary with respect to the speed of the wheels or the transmission of the vehicle. Making use of basic physical properties, a small variation of the original design, allow control of the distance and acceleration of the vehicle ${ }^{7}$. 


\subsection{Sensors by Hall Effect}

The measurement technology by Hall effect is one of the most effective methods and used for the indirect measurement of many physical phenomena at present. Although its discovery dates back to 1879 , it was not until the late 1980s and early 1990s that its use could be massified thanks to the mass production of the electronics needed to easily condition its signals. The Hall Effect was discovered by Dr. Edwin Hall while presenting his candidacy for a doctorate at John Hopkins University ${ }^{\circ}$.

Depending on the manufacturer and other design considerations such as vehicle type, types of transmission or purpose, there are several configurations available to measure the speed of a vehicle by means of Hall Effect sensors. In the designs proposed by the various manufacturers, regardless of their location, they all use the same principle of operation. The majority of configurations of speed sensors by Hall Effect have as active element a toothed wheel or pinion of some ferro-magnetic material, which allows generating the precise configuration for the measurement of electrical pulses according to the angular velocity of some mechanical element associated to the movement of the vehicle. Within the integrated circuit, they contain filtering capacitors and a powerful polarization magnet, inside a sealed non-magnetic packaging that protects the electronics from aggressive environmental conditions?

\subsection{Sensors by Satellite Technology}

Currently, navigation systems have been converging to receivers with the capacity to calculate the mobile speed vector on land and sea, obtaining information from several parallel location networks, known as Global Navigation Satellite Systems. Which are particularly common in the market those who use Navstar navigation networks promoted by the United States Department of Defense and the Glonass navigation system sponsored by the Russian space agency. It can be shown mathematically that the basic measurements that can be made from a signal transmitted by a satellite are its distance and relative speed. These measurements can be used to derive navigation information from a satellite if they are observed for a sufficient period of time or by measuring several satellites simultaneously 10 .

The basic principle of the GPS system is based on determining the location of the mobile with respect to the satellite $\vec{R}_{\mathrm{m}}$. Once the trajectory and the law of motion are known, by means of the equations of the satellite dynamics $\vec{R}$, the position of the mobile will be determined by the difference as shown in Equation 10.

$$
\vec{R}=\vec{R}_{s}-\vec{R}_{m}
$$

To determine $\vec{R}_{\mathrm{m}}$, the satellite constantly transmits 2 L-band signals with a code to determine the distance and a navigation message. If the distance to a single satellite were known, the locus where the mobile would be located is a sphere; if the distance to two satellites were known, the probable locus would be a circumference and if the known distances were 3 , the locus will be the intersection of one or two points, one of which can be rejected by estimation.

If the satellites and the mobile were perfectly synchronized in time, the mathematical problem would be reduced to solve three equations with three unknowns, but as it generally does not happen in this way for relativistic, ionospheric, geographic and electronic effects, a new unknown generated by the movement of the mobile clock; thus a measurement of a fourth satellite or some more, reduces these effects in the accuracy of the measurement, being again configured a system of four equations with four unknowns. Since an error is always presumed in the clock, each measure of distance will be displaced some meters/centimeters ${ }^{11}$.

\subsection{Sensors by Doppler Effect}

Although navigation by Doppler effect has not been a common technique in land transport vehicles, it is one of the most precise techniques available on the market for the measurement of distance, speed and acceleration parameters of a mobile, however, due to its implementation costs, as of the date of this study is only reserved for high-end vehicles such as accident prevention system (CAS, Collision/Crash Avoidance Systems) and some applications of precision agriculture. In Figure 4 one can see a typical configuration diagram for a speed sensor based on Doppler Effect. 


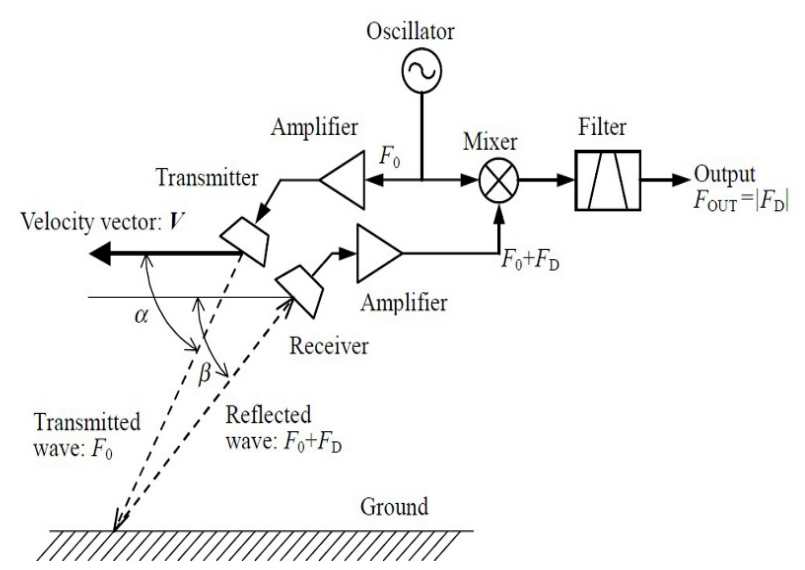

Figure 4. Estimation of the position of a mobile.

An oscillator generates a standard signal of frequency $\mathrm{F}_{0}$, this signal is amplified and transformed into radiant energy by means of a transmitter that directs it in a diagonal trajectory at an angle towards the moving surface of the mobile. The energy wave is reflected by the movement surface and separated into several components, some of which are detected by a receiver. Classical physical theory speaks of frequency displacement due to the effect of the path of a wave in space, known as the Doppler Effect. Since $F_{D}$ is the frequency shift due to the Doppler Effect, the signal received by the system will be $\mathrm{F}_{0}+\mathrm{F}_{\mathrm{D}}$. The system will receive this signal and combine it electronically with the original signal. By means of low pass filters, allowing the system to have a $\mathrm{F}_{\text {out }}$ output signal that will be equivalent to the frequency shift of the signal by Doppler Effect.

The output frequency is proportional to the transmission frequency, the speed of the vehicle and other parameters known as the emission and reception angles of the signal. By clearing $\mathrm{V}$, an instrument for measuring the absolute speed of the mobile device can be built with great performance and independence of external parameters.

\subsection{MEMS Sensors (Accelerometers)}

Accelerometers are devices for measuring acceleration, vibration and consequently almost any dynamic variable of a mobile by means of appropriate mathematical and physical arrangements. These devices convert the acceleration of gravity or movement into an analog electrical signal proportional to the force applied to the system, or mechanism subjected to vibration or acceleration. This analog signal indicates in real time the instantaneous acceleration of the object on which the accelerometer is mounted. An accelerometer measures the inertial force generated when a mass is affected by a change in velocity. This force can vary the tension of a spring, deform an element, or modify the frequency of vibration of a mass. Accelerometers are directional, to monitor acceleration in three dimensions are used multi-axis accelerometers (axes $\mathrm{x}, \mathrm{y}, \mathrm{z}$ ).

The basic operating principle of accelerometers is the detection of force exerted on a mass with elastic limitation. Consider a simple mechanical system, consisting of a fixed mass $\mathrm{m}$, with a spring with a stiffness $\mathrm{k}$ (constant). If the mass moves a distance $\mathrm{x}$, the acceleration due to the restoring force of the spring is $\mathrm{F}=\mathrm{k}^{*} \mathrm{x}$. Substituting in Newton's equation, we find that $\mathrm{a}=\mathrm{k}^{*} \mathrm{x} / \mathrm{m}$ and we can derive the magnitude of the acceleration by observing the motion $\mathrm{x}$ of the fixed mass. This fundamental principle is used even in the most sophisticated and cost-effective electromechanical accelerometer, and even in the modern micro-mechanized accelerometer ${ }^{12}$.

Particularly in the automotive industry the use of accelerometers is widely spread in safety, comfort and vehicle control applications. Essentially there are two important challenges to contemplate the use of these devices with navigation objectives. First of all, the alignment of the accelerometer axes with the axes of the vehicle is a crucial variable that many designers do not always achieve in an effective and stable way over time. The second condition is that as an autonomous navigation device it has a high dependence on the calibration of the initial point of speed and position. In this way and due to the phenomenon of integration of the error in time, often these devices are used in conjunction with others to help maintain the initial calibration point, and only work autonomously when the main navigation device is not found available (dead reckoning navigation).

\section{Factors that Affect the Accuracy of Automotive Speedometers}

Although there are numerous technologies for measuring the distance traveled and the speed of a vehicle, the most basic are those that predominate in the automotive market, with a special predominance of speed sensors for parasitic loads and Hall Effect. Generally, the system is based on the rotation of some element of 
the motor train of the vehicle, which is transformed into a signal that goes directly to the vehicle's visualization or in the best of cases to a central control system, also called ECM (Engine Control Unit), where the variables of distance traveled and vehicle speed are presented to the user through analog or digital interfaces.
By means of a standard of the American Society of

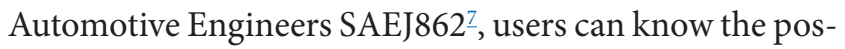
sible inaccuracies to which these measurement elements may be subjected, due to different external factors that in many cases are difficult to control by vehicle manufacturers, which are summarized in the Figure 5:

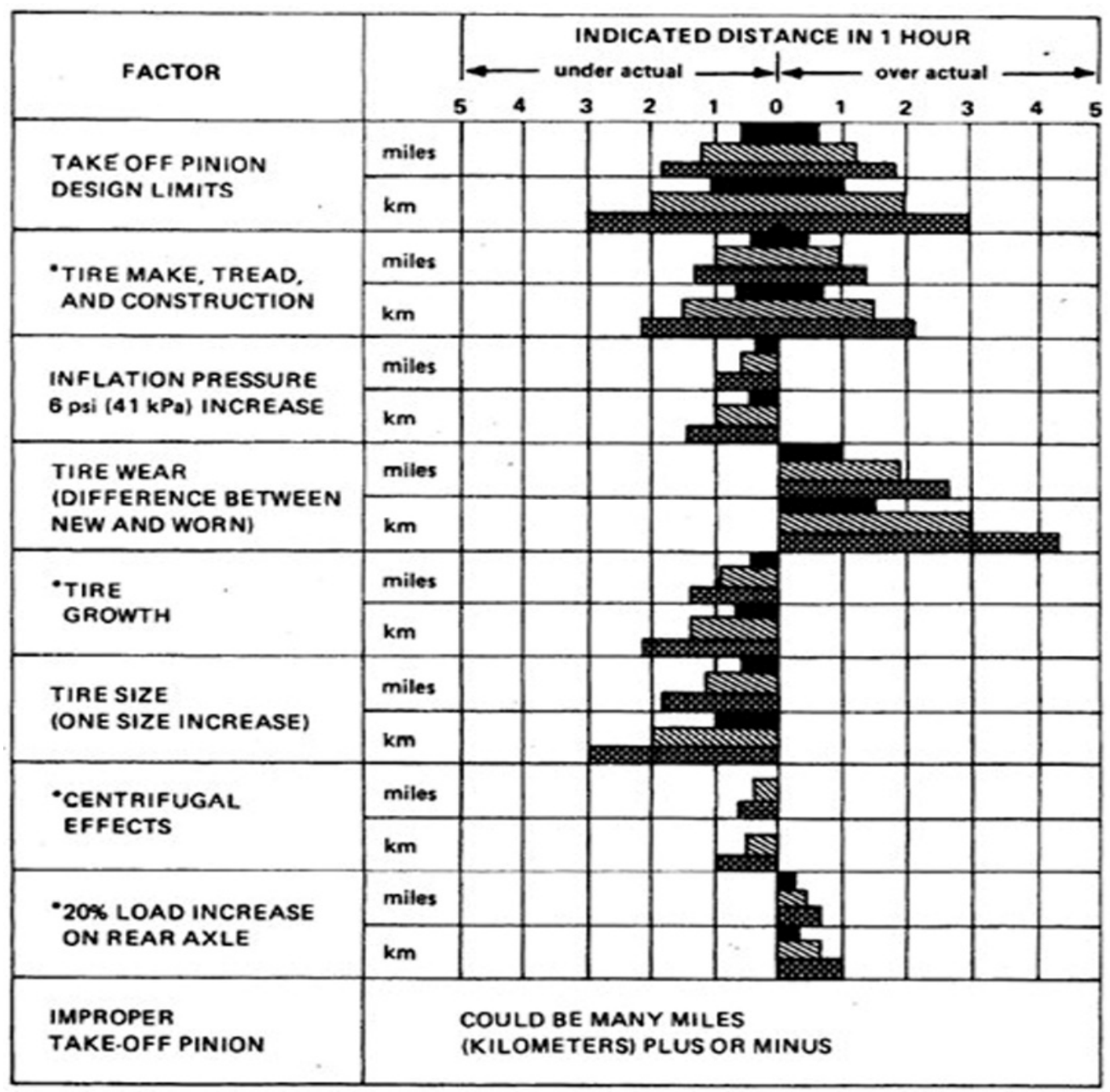

VEHICLE SPEED AT:

$30 \mathrm{mph} / 48 \mathrm{~km} / \mathrm{h}$

$60 \mathrm{mph} / 96.5 \mathrm{~km} / \mathrm{h}$

$90 \mathrm{mph} / 145 \mathrm{~km} / \mathrm{h}$

-VAlues shown are for bias ply tires. Radial and BELTED TIRES ARE AFFECTED A LESSER AMOUNT.

Figure 5. Summary of possible causes of inaccuracy in a speed meter ${ }^{7}$. 


\section{Protection Meter of Acceleration and Speed}

The main objective of the prototype will be to check that the technological elements included inform, in a preventive way to the driver, when some of the kinematic variables of the vehicle is getting out of control for him to apply the corrective measures before the errors lead to an accident of unpredictable consequences. With the above, the idea is finally to involve the operator in the correction of their bad practices through the promotion of the selfcontrol and self-care culture behind the wheel, for which the system shown in Figure 6 is proposed.

The methodology used is simple. Each variable analyzed is assigned a value from 0 to 100 , where 100 is the technology that best meets expectations and 0 the worst performs, in the end the scores are averaged to know the sensor with the best characteristics for the design.

As can be seen in Table 1, there are many aspects that determine the ideal selection of the speed sensor, depending on whether you are looking for ease of installation, design time, precision, useful life, among others, a sensor or other it may be the optimum for a particular design.

To weigh the benefits of the systems, in Table 2 the main characteristics of these technologies are related in a standardized way in order to make an optimal sensor decision for the prototype.

As you can see, sensors based on global satellite navigation systems take precedence over their competitors because of their balance between different key design and implementation variables. This type of sensors is easily integrated by means of fast development platforms such as microcontrollers and thanks to its wide diffusion in the market, the acquisition and maintenance costs of this technology make it ideal for the segment of users that we want to impact. On the other hand, the independence of the vehicle's mechanical components makes it ideal for design.

The choice of the GNSS module as a main element brings with it many other benefits in the design, construction and installation of the prototype, since a large part of the algorithmic challenges are solved in commercial chips such as the Ublox6 module ${ }^{\underline{13}}$ used in the prototype, which With a serial connection, with low-cost processors, information is used to implement the necessary algorithms to generate the necessary alerts that were implemented with standard LCD display modules, amplified audio players and standard low-cost keyboard inputs. The final result of the integration of all the components can be seen in the designed PCB shown in Figure 7.

Once designed, simulated and tested in the laboratory, the prototype is manufactured, which can be seen in Figure 8.

\subsection{Field Tests}

According to the World Health Organization, above $30 \mathrm{~km} / \mathrm{h}$, the probability that a traffic accident involving a walker person ends as a fatal accident is $10 \%$, rises steeply until it reaches almost $90 \%$ probability of death when the accident occurs at only $50 \mathrm{~km} / \mathrm{h}$, speed easily exceeded in any city road as shown in Figure $9 \frac{14}{}$.

Verification tests of the operation of the system under uncontrolled conditions were carried out through the installation of 3 prototypes in vehicles with different characteristics, where the aim was to validate the functionality of the system under limit conditions. Three vehicles with different driving characteristics were chosen, as well as different characteristics of the terrains they traveled to guarantee the universality of the tests. The vehicles chosen were: Chevrolet Optra, Nissan Frontier, and International Truck.

Table 1. Comparative analysis of existing technologies for speed measurement

\begin{tabular}{|l|l|l|l|l|l|}
\hline 1.1 & Electro mechanics & Hall Effect & GNSS & MEMS & Doppler Effect \\
\hline Hardware unit cost & 50 USD & 70 USD & 100 USD & 300 USD & 500 USD \\
\hline Cost design & $\begin{array}{l}25 \text { hours } \\
2000 \text { USD }\end{array}$ & $\begin{array}{l}50 \text { hours } \\
4000 \text { USD }\end{array}$ & $\begin{array}{l}50 \text { hours } \\
4000 \text { USD }\end{array}$ & $\begin{array}{l}50 \text { hours } \\
4000 \text { USD }\end{array}$ & $\begin{array}{l}150 \text { hours } \\
12000 \text { USD }\end{array}$ \\
\hline Prototype manufacturing cost & 500 USD & 1500 USD & 500 USD & 2000 USD & 2500 USD \\
\hline Installation cost & 250 USD & 250 USD & 50 USD & 100 USD & 400 USD \\
\hline Annual maintenance cost & 50 USD & 50 USD & 25 USD & 25 USD & 300 USD \\
\hline Spatial resolution & $100 \mathrm{~m} / \mathrm{km}$ & $75 \mathrm{~m} / \mathrm{km}$ & $12 \mathrm{~m} / \mathrm{km}$ & $8 \mathrm{~m} / \mathrm{km}$ & $4 \mathrm{~m} / \mathrm{km}$ \\
\hline Temporary resolution & $1000 \mathrm{~ms}$ & $1 \mathrm{~ms}$ & $100 \mathrm{~ms}$ & $0.1 \mathrm{~ms}$ & $0.01 \mathrm{~ms}$ \\
\hline
\end{tabular}


Table 2. Relative analysis of existing technologies for speed measurement, with standardized scores

\begin{tabular}{|l|c|c|c|c|c|}
\hline 1.2 & Electro mechanics & Hall Effect & GNSS & MEMS & Doppler Effect \\
\hline Hardware unit cost & 100 & 75 & 50 & 25 & 0 \\
\hline Cost design & 100 & 75 & 75 & 75 & 0 \\
\hline Prototype manufacturing cost & 100 & 75 & 100 & 25 & 0 \\
\hline Installation cost & 50 & 50 & 100 & 75 & 0 \\
\hline Annual maintenance cost & 75 & 75 & 100 & 100 & 75 \\
\hline Spatial resolution & 0 & 25 & 50 & 75 & 100 \\
\hline Temporary resolution & 0 & 25 & 50 & 75 & 200 \\
\hline
\end{tabular}

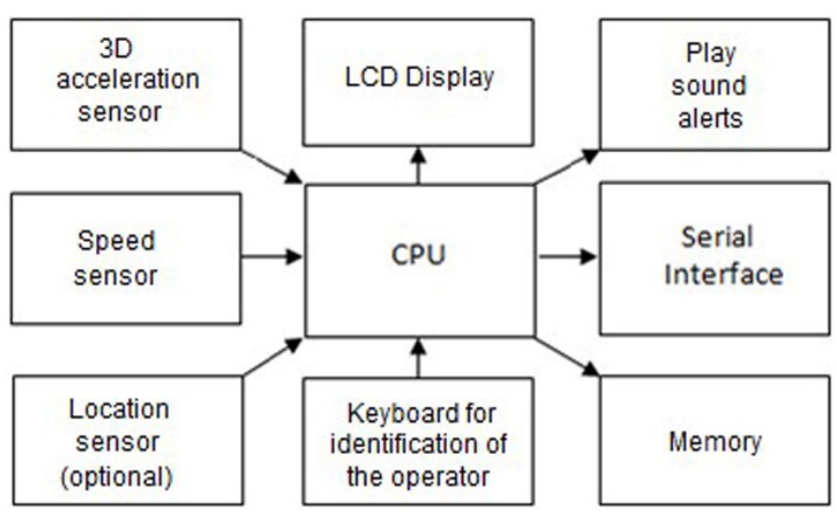

Figure 6. Block diagram of the prototype.

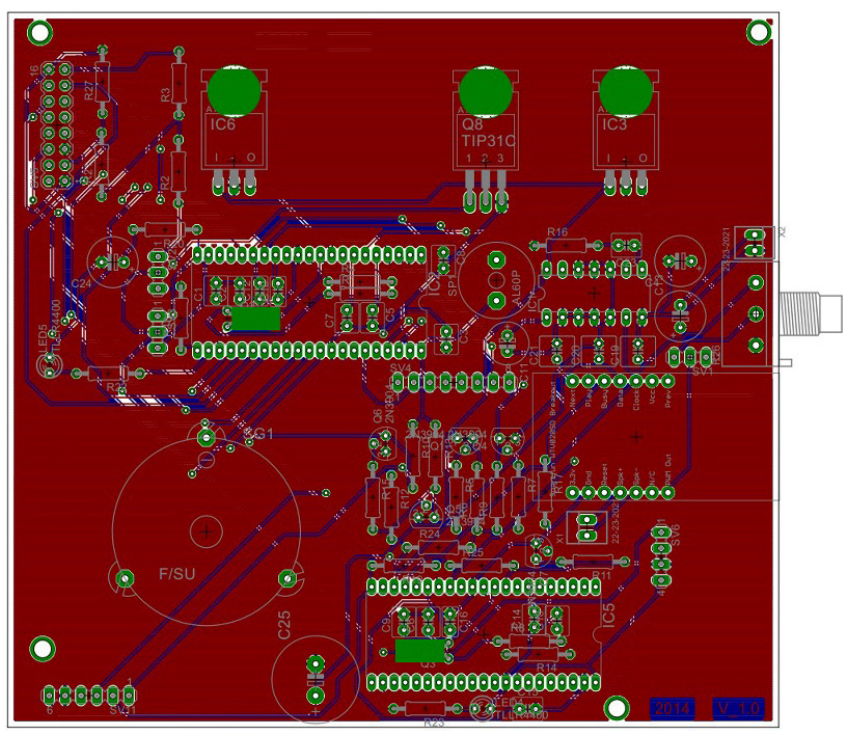

Figure 7. Prototype PCB.

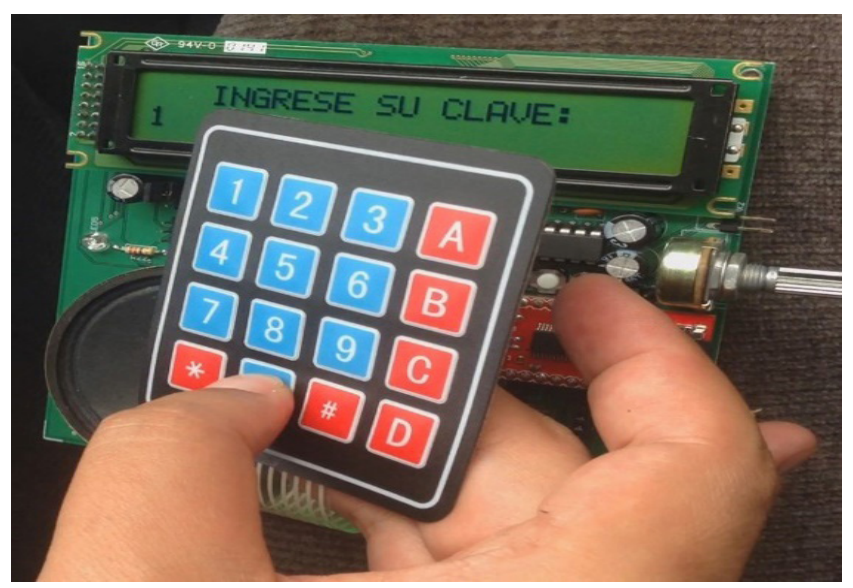

Figure 8. Image of the prototype implemented,

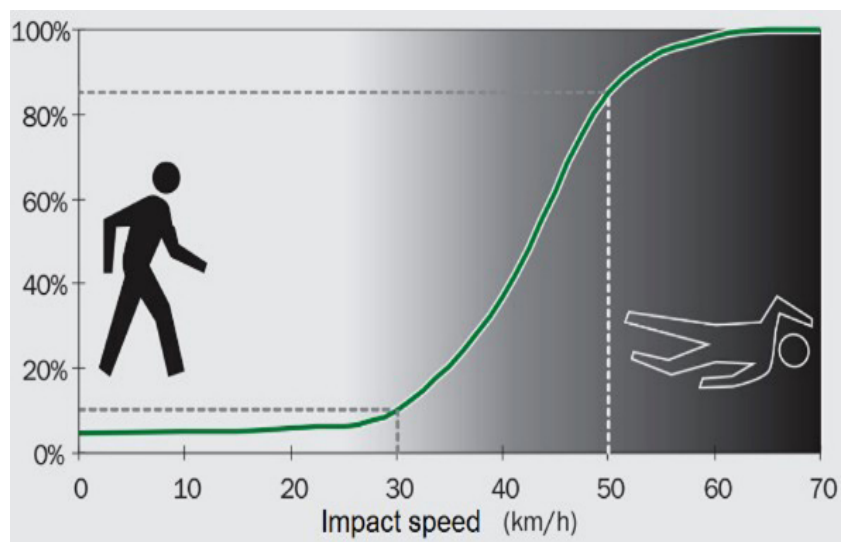

Figure 9. Probability of fatal injury to a walker colliding with a vehicle ${ }^{14}$. 


\subsection{Family Vehicle Test Drive}

With a journey made during 30 days in the city of Bogota (Figure 10), the family vehicle shows an ideal behavior for this study, where $80 \%$ of the time the vehicle makes urban trips, and only in 4 days it makes trips out of the city, behavior very similar to the typical use of an average family vehicle. The analysis of the speeds reached by the vehicle is also consistent with the expected values, since by reviewing the history of speeds recorded by the vehicle, the behavior is characterized by large jumps between rest up to urban speed limits and from there again to rest in very short time intervals, which supposes a high stress regime for both the driver and the vehicle, having to force the human body and the machine to constant accelerations and braking, with the corresponding risks that each of these actions takes.

In order to establish a quantitative measurement of the behavior of this vehicle, the American standard of operator qualification will be used, which allows assigning an ascending index of average number of faults $\mathrm{x}$ kilometers traveled to establish an objective profile of aggressiveness of the driver, in this way. There is an index that evaluates the frequency which a vehicle operator deviates from safe behavior. According to the same standard, an average driver should be below 5 faults per 100 kilometers, a driver with training needs would be in the range of 5 to 10 faults and a high risk driver would be above 10 faults per 100 kilometers ${ }^{15}$.

This analysis is one of the main achievements of the device object of this study, since the obtained data present an objective tool to determine the behavior of the driver and establish strategies to mitigate risks. The average score of fouls in the period of analysis is 4.7, which is close to the appropriate driving limit value of the vehicle, in addition, the appearance of 58 excess speed in the month and 59 sudden accelerations would require an immediate preventive action to avoid the aggressive driving of this driver.

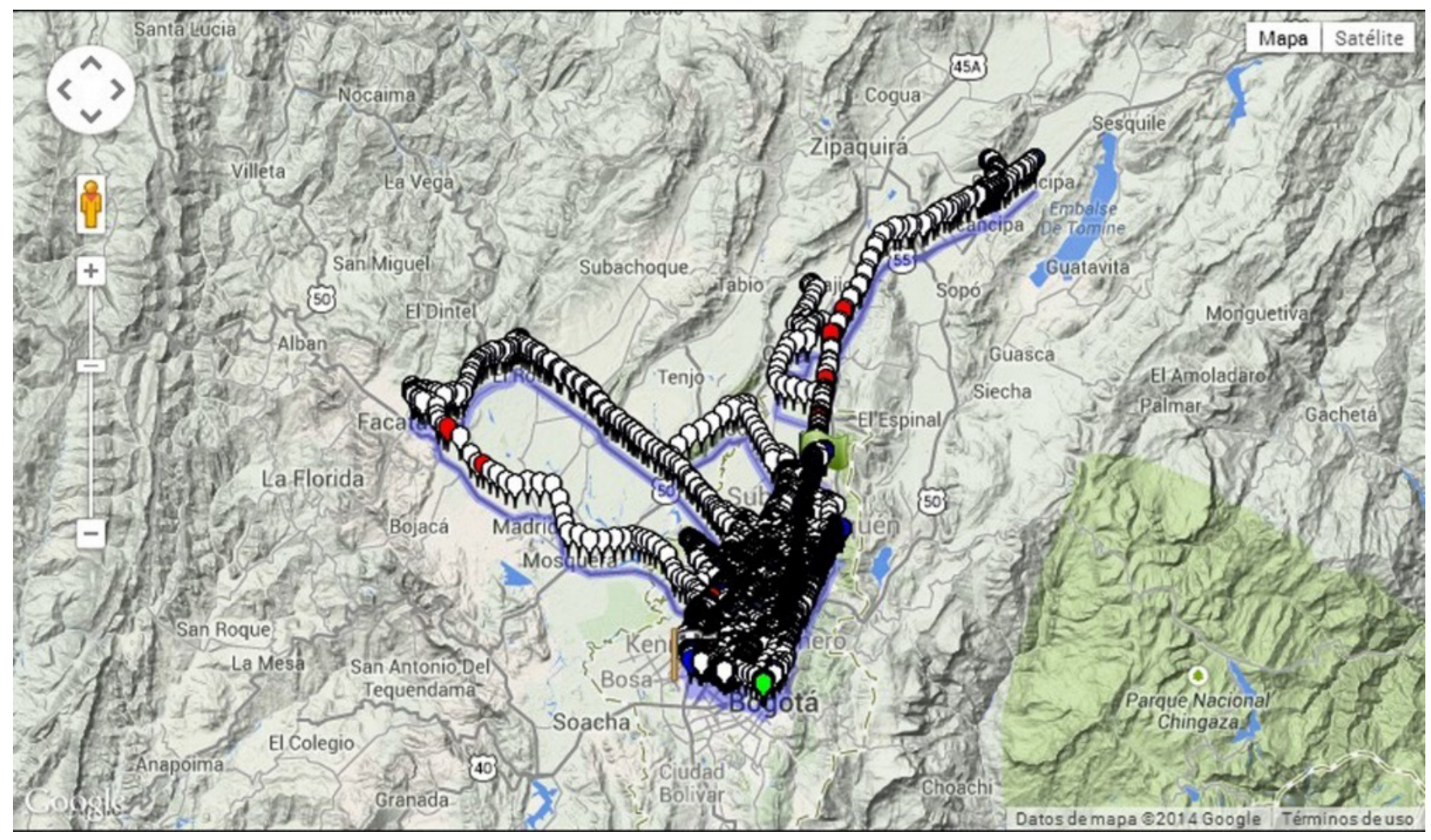

Figure 10. Viewing information obtained from the family vehicle using Google Maps tool. 


\section{Conclusions}

In the absence of clear policies in the standardization of road safety, it is a function and duty of private industry, carriers, cargo generators, owners of private vehicles and, why not, the academia, ensure the prevention and mitigation of bad practices that lead to traffic accidents, an aspect in which this research aims to provide knowledge through an electronic device that effectively alerts operators when any of the key dynamic variables for the control of the vehicle is going beyond its limits, derived from some conscious or unconscious action in the operation of the vehicle, thus allowing us to generate a habit of self-care and self-control in our drivers and thus achieving in the medium term, with the accompaniment of a process control of the state and the industry, a significant decrease in the accident and mortality rates.

\section{Acknowledgments}

The authors thank the Universidad Distrital Francisco Jose de Caldas, the LASER research group and the WaveComm Corporation that supported the development and testing of the project.

\section{References}

1. Anuario estadistico de accidentalidad vial en Colombia. Universidad de los Andes, Corporacion fondo de prevencion vial. 2011. p. 1-143.

2. Peden M, Taroyan T. Informe sobre la situacion Mundial de la Seguridad Vial. Organización Mundial de la Salud; Ginebra, Suiza. 2009.

3. Audi. 2018. https://en.wikipedia.org/wiki/Audi.
4. Wong JY. Theory of ground vehicle. 1st Ed. John Wiley and Sons. 1978.

5. Quan Y, Shenjun T, Kaifeng L, Yibing L. Investigation and analysis of drivers speedometer observation and vehiclespeed cognition. Fifth International Conference on Measuring Technology and Mechatronics Automation; 2013. p. 667-70. PMid: 23975370. https://doi.org/10.1109/ICMTMA.2013.166.

6. An X, Xiangming Q. Research on distributive regularity of vehicle speedometer indicated error and its modeling. International Conference on Optoelectronics and Image Processing. 2010; 2:214-7. https://doi.org/10.1109/ ICOIP.2010.43.

7. SAE ARP4754A. Guidelines for development of civil aircraft and systems, the Engineering Society for Advancing Mobility Land Sea Air and Space. Aerospace Recommended Practise; Warrendale, USA. 2010.

8. Sriratana W, Murayama R, Tanachaikhan L. Application of the HES in angular analysis. Journal of Sensor Technology. 2012; 2(2):87-93. https://doi.org/10.4236/jst.2012.22013.

9. Ramsden E. Hall-Effect sensors: Theory and Application. 2nd Ed. Elsevier. 2006. p. 23-40.

10. Hollreiser M, Crisci M, Sleewaegen JM, Giraud J, Simsky A, Mertens D, Burger T, Falcone M. Galileo signal experimentation. GPS World. 2007. p. 37-44.

11. Corbasí A. Sistemas de navegacion: Desde el compás magnetico a la navegaciin por satelite. McGraw-Hill Interamericana de Espa-a. 1998.

12. A (not so) short introduction to MEMS. 2016. https:// memscyclopedia.org/introMEMS.html.

13. U-blox 6 Receiver Description. Including Protocol Specification. Revision for FW 7.03. 2013. p. 1-222.

14. World Health Organization. Control de la velocidad: Un manual de seguridad vial para los responsables de tomar decisiones y profesionales. Ginebra, Sociedad Global de Seguridad Vial (GRSF). 2008. p. 1-266.

15. Driver Qualification Handbook, NSW. Transport Roads and Maritime Services. 2013. 